


\title{
Geodinámica en relieves montañosos: estudio de la Reserva Biológica Alberto Manuel Brenes, San Ramón, Costa Rica
}

\author{
Geodynamic in mountain reliefs: study of the Alberto Manuel Brenes Biological Research, San Ramón, \\ Costa Rica \\ Marvin Quesada Quesada ${ }^{1}$
}

Recibido: 4/4/2016 / Aprobado: 1/11/2016

Resumen

\begin{abstract}
Se analiza la formación geológica de la Reserva Biológica Alberto Manuel Brenes (ReBAMB), la cual se originó inicialmente por un vulcanismo explosivo caracterizado por la emanación de flujos lávicos piroclásticos. Existen rocas ígneas y sedimentarias, en su mayor parte terciarias, en tanto que los depósitos cuaternarios son producto de la erosión y meteorización de las rocas más antiguas. Las estructuras geológicas presentan formas distintivas, con fallas geológicas, así como formas caldéricas producto de la explosión o colapsos volcánicos. La topografía muestra condiciones litológicas caracterizadas principalmente por aparatos volcánicos con lavas, domos, calderas volcánicas, cráteres y formas de erosión diferencial. Similarmente, existen cuerpos intrusivos granitoides y rocas sedimentarias, localmente meteorizadas, así como lavas de posibles cuerpos subvolcánicos subordinadas en la secuencia estratigráfica. El valor de amenaza sísmica sugerido es del orden de 4.0 a $4.5 \mathrm{~m} / \mathrm{s}^{2}$.
\end{abstract}

Palabras claves: Costa Rica, ReBAMB, vulcanismo, flujos piroclásticos, rocas ígneas y sedimentarias, intrusivos.

Abstract

The geological formation of the Manuel Alberto Brenes Biological Research is analyzed because there are igneous and sedimentary rocks that belong to the Tertiary period, while some old rocks are product of erosion $n$ weathering during the Quaternary period. The geological formation was originated at the beginning for explosive volcanism characterize for pyroclastic lavas. The geologic structures present different forms, like falls and calderas as a consequence of explosive volcanoes. The topography shows lithological conditions characterize mainly for volcano forms like lavas, domes, calderas, craters and different erosional landforms. Similarly, there are intrusive and sedimentary rocks with some degree of weathering and lavas coming from sub volcanic activity. The suggested seismic risk is between 4.0 and $4.5 \mathrm{~m} / \mathrm{s}^{2}$.

Key words: Costa Rica, ReBAMB, volcanism, pyroclastic rocks, igneous rocks, sedimentary rocks, intrusive.

\section{Introducción}

Una interpretación geológica de la Reserva Biológica Alberto Manuel Brenes (ReBAMB) permitirá conocer la evolución geodinámica de una parte de la cordillera de Tilarán, además permitirá establecer una metodología de investigación muy útil en el campo geográfico o de otras disciplinas que tienen interés de estudiar formaciones geológicas. La ReBAMB es una región parte de una zona sísmica tectónicamente activa y con procesos erosivos y depositacionales de materiales terrosos, coluviales y aluviales importantes, producto del abrupto relieve, clima lluvioso, cálido, con alta meteorización de las rocas, lo cual da como resultado suelos muy frágiles. Los datos geológicos serán particularmente importantes para ejecutar planes de acción, con planificación estratégica y protección de los recursos hídricos.

\footnotetext{
${ }^{1}$ Profesor catedrático, Departamento de Ciencias Naturales, Sede Occidente, Universidad de Costa Rica. Correo electrónico: marvin.quesada@ucr.ac.cr
} 
Los estudios geológicos en Costa Rica se han realizado principalmente en los sectores donde se registra una mayor actividad volcánica o sísmica, tal es el caso de la sección de la cordillera volcánica Central y el complejo de Nicoya. En tanto, aquellas regiones de escasa actividad volcánica reciente, como es el caso de la sección de la cordillera de Tilarán, son escasas las investigaciones, dataciones y descripciones detalladas de su geodinámica. Sin embargo, la fuerte actividad sísmica registrada en Costa Rica durante el 2012 (5 de septiembre y 23 de octubre) provocan que esta región sea de gran interés, dada la cercanía con Guanacaste, sitio donde ocurrieron los últimos eventos sismos importantes en el país, con magnitudes de 7.6 y 6.6 en la escala Richter, respectivamente. Asimismo, cuando ocurren sismos de tal magnitud suelen reactivarse aparatos volcánicos y fallas, tal es el caso de los volcanes Turrialba, Poás y las fallas en lugares como Zarcero, Escazú y Tobosí, en San José y Cartago, respectivamente.

La sección este de la Cordillera de Tilarán podría constituir una amenaza volcánica, al existir varias geoformas volcánicas que presentan condiciones propicias para que tal condición se reactive o se desarrolle. Similarmente, algunos sectores cercanos a la Reserva han sido propuestos como campo geotérmico, tal es el caso de Pocosol de San Carlos (Vargas, 2001), lo que sugiere que la cámara magmática es superficial. En regiones vecinas se han hecho estudios que muestran muy buenos resultados, tal es el caso de la cuenca superior del río Peñas Blancas en el cantón de San Ramón (Vargas, 2001).

\section{Área de estudio}

Gran parte de la Reserva Biológica Alberto Ml. Brenes (ReBAMB) se ubica en la parte superior de la cuenca del río San Lorenzo. Un 90\% de su superficie se localiza en el distrito de Los Ángeles del cantón de San Ramón (provincia de Alajuela) y el resto en el distrito La Unión del cantón de Montes de Oro (provincia de Puntarenas). Por consiguiente, la ReBAMB abarca 7800 hectáreas y constituye cerca del $7 \%$ del territorio ramonense (figura 1).

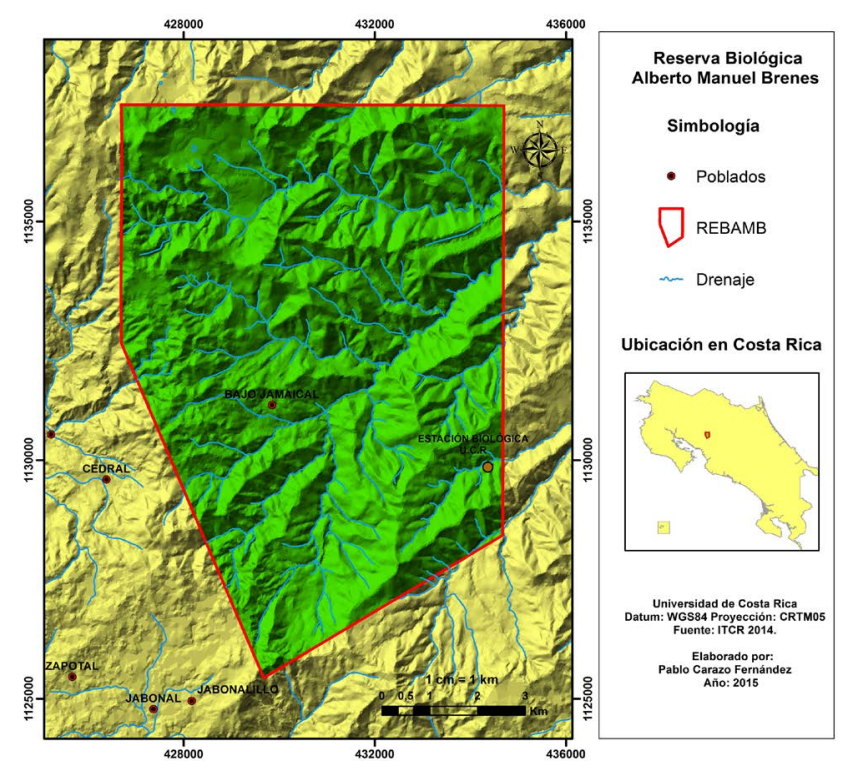

Fig. 1. Ubicación de la REBAMB, San Ramón, Costa Rica.

El sector donde se ubica fue declarado como Área Silvestre Protegida el 10 de junio de 1975, mediante el Decreto ejecutivo no. 4950-A de junio de 1975, en la categoría de manejo de Reserva Forestal y porque la mayor parte se encuentra en el cantón de San Ramón, se le nombró Reserva Forestal de San Ramón. En 1991 fue redefinida como Zona Protectora de este cantón, mediante el Decreto ejecutivo no. 20172-MIRENEM de 1991. No obstante, el 20 de agosto de 1993, de acuerdo con la Ley No. 7354 del 10 de agosto de 1993, publicada en el diario oficial La Gaceta no. 159, se modificó a Reserva Biológica Alberto Ml. Brenes (Salazar, 2000).

El acceso a la ReBAMB es posible por el sector Caribe a través de la vía a Bajo Rodríguez hasta el sitio denominado Los Lagos, a partir de allí se toma una vía con lastre y rodeada de bosque. En lo interno de la Reserva solo se puede ingresar por los senderos construidos por los funcionarios de la Sede de Occidente que laboran en la estación, la cual recibe y alberga estudiantes, docentes e investigadores, quienes reciben charlas o hacen investigaciones. En general, el acceso es difícil la mayor parte del año, no solo por las condiciones atmosféricas sino también por lo abrupto del relieve. 
El recorrido y la visualización de esta región son difíciles en el terreno, por lo que parte de la investigación se realizó mediante el uso de fotografías aéreas. Durante la mayor parte del año es una zona lluviosa, por lo que la vegetación es sempervirente. En tanto, los ríos, confluencias, tributarios y colectores cubren gran parte del territorio, de forma que mantienen no solo un caudal constante sino una densa red de drenaje. Además, es una zona muy abrupta desde el punto de vista topográfico, producto del tectonismo y el vulcanismo.

\section{Métodos}

La metodología aplicada en esta investigación se basa en métodos tradicionales del campo geológico, como son: recopilación de información, trabajo de campo, fotointerpretación, cartografía topográfica y geológica. Se identificaron y se cartografiaron las formaciones geológicas presentes en la reserva en estudio.

En las primeras dos giras, el trabajo de campo consistió en reconocer de manera general la Reserva. En las siguientes tres giras se recorrió varias veces la zona, para visualizar, identificar y tomar fotografías, sobre unidades geológicas, afloramientos, rocas, taludes, deslizamientos, dirección de los ríos, pendientes, serranías, lagunas, granulometría de los lechos fluviales, entre otros aspectos, así como corroborar la cartografía preliminar. Como parte de los levantamientos de terreno el principal foco de atención fueron aquellos sitios que presentaban ciertas características sobresalientes, como eran su estado de meteorización, dirección de los estratos, tipos de rocas, la presencia de drenajes, entre otros. Se utilizó un equipo de posición satelital Garmin 12 para localizar geográficamente algunos de estos sitios.

En síntesis, para realizar la investigación se recopiló la información, tanto del campo como de bases de datos existentes sobre el área en estudio. Cuando no se contó con suficiente información se utilizaron fotografías aéreas, de las cuales se realizó una fotointerpretación geomorfológica. Se procesó la información y se realizó una cartografía preliminar.
En la elaboración de los mapas se utilizó cartografía digital empleando el programa ArcView 3.9. Con las unidades descriptivas definidas se procedió a completar y elaborar una cartografía geológica básica y un mapa de la geología estructural de la ReBAMB. Por último, caracterizaron las unidades geológicas, tectonismo y la red de drenaje, así como la evolución de los procesos formadores de la Reserva en estudio. Adicionalmente, se determinaron las direcciones y longitudes de los alineamientos tectónicos con el fin de ser representados cartográficamente.

Por el hecho de ser una reserva bajo cobertura forestal muy densa, sin caminos secundarios, sin explotaciones de tajos y minería. Los suelos se encuentran cubiertos de biomasa, una vegetación muy densa y con precipitaciones durante la mayor parte del año. Por otro lado, es un sitio de difícil acceso, no solo por la mínima existencia de caminos y senderos, sino también por la irregularidad del terreno. Esto se vuelve un obstáculo para hacer los diferentes reconocimientos en el campo. Por lo tanto, se dispuso de los pocos accesos posibles como son el camino principal, algunos senderos, ríos y quebradas.

\section{Marco geológico regional}

El sur de Nicaragua, toda Costa Rica y la sección occidental de Panamá, los cuales conforman la América Central Meridional, se formaron hace 120 millones de años. Según Lloyd (1963) y Castillo (1984) le llamaron el Orógeno Sur Centroamericano, lo que indica que es el territorio más joven de las Américas. Sin embargo, desde hace unos 150 millones de años ya se iniciaban las primeras interacciones tectónicas y volcánicas gestoras del territorio de Costa Rica, lo cual se manifestó a través de un conjunto de islas a las que se les denominó el arco externo (Bergoing, 1988). Dicho proceso tuvo lugar desde el fondo marino, dado que en ese momento existía un canal interoceánico, al que se le llamó Canal Centroamericano. En este canal tuvo lugar una intensa actividad volcánica submarina, como consecuencia de las fuertes presiones a la que se había sometido el fondo oceánico, desde el interior de la tierra. Es así como se formó un arco de 
islas volcánicas, entre el Jurásico y el Cretácico, hace 140 millones de años. Es el proceso de subducción de la Placa Coco por debajo de la Caribe a lo largo de la fosa Mesoamericana, en el Océano Pacífico, el cual se ha encargado de formar gran parte del territorio de Costa Rica (Cortés, 1994).

En el Plioceno Tardío y en el Cuaternario temprano hubo períodos de un vulcanismo explosivo, caracterizado por magmas someros diferenciados de tipo dacítico y riodacítico (Vargas, 2001). Weyl (1956) señala que dicho vulcanismo produjo extensos depósitos de flujos piroclásticos, asociados con estructuras caldéricas de colapso. Este tipo de intrusiones magmáticas originaron las anomalías térmicas observadas, especialmente en la sección de la cordillera de Tilarán. Vargas (2001) describió la geología de la cuenca alta del río San Lorenzo (cuenca en la que se ubica la ReBAMB) e indicó que pertenece al vulcanismo de formación Aguacate, de finales de la fase orogénica, esta geología se manifestó desde el Plioceno (Terciario) y a la vez constituye una zona de contacto con el vulcanismo del Plioceno Cuaternario de la cordillera de Guanacaste y de la cordillera Central.

La sección de la cordillera de Tilarán es un cinturón montañoso de origen magmático, alineado de NW- SE, situado al SW a lo largo del Arco Interno del Orógeno de Costa Rica del eje volcánico activo, pero en una posición de antearco, o sea hacia el Pacífico, con respecto a las secciones de las cordilleras de Guanacaste y Central (Dengo, 1962). Desde el punto de vista morfológico no se reconocen aparatos volcánicos muy recientes, solamente algunos edificios en proceso erosivo (Vargas, 2001), como consecuencia se ha formado una topografía muy abrupta.

Existe una desviación de la cordillera hacia el sur donde se ubican los Montes del Aguacate, que terminan en Puriscal. Por el sector norte se ubican los cerros del Hacha, San Roque, Cañas Dulces y Gorgona que son parte del Aguacate y los cuales son considerados relictos volcánicos. Por lo tanto, esta cordillera está formada principalmente por rocas volcánicas, originadas durante el periodo Mioceno. Schaufelberger (1935), citado por Chaves y Sáenz (1974), la definió como un horst limitado por las fallas de las Juntas y del Arenal. De acuerdo con Weyl (1956), la cordillera de Tilarán tiene una historia geológica con la siguiente secuencia: vulcanismo inicial, plutonismo sin orogenia y vulcanismo subsiguiente. Dengo (1962) ya había expuesto esta secuencia, quien supuso una actividad eruptiva durante el Eocénico y otro más reciente con su máxima actividad durante el Mioceno, con cierta actividad intrusiva intermedia. En síntesis, todos los investigadores antes mencionados concuerdan que la orogénesis principal de la cordillera de Tilarán se da durante el Mioceno, la cual se basó en actividad volcánica.

\section{Geología local}

Los relieves volcánicos, a diferencia de las emanaciones plutónicos, son superficiales y determinan la evolución morfológica de una región, pueden ser plegados, fallados o fosilizados por una transgresión marina. Las coladas de lava sellan el relieve que recubren poniendo en evidencia las topografías antiguas (Alvarado, 2000). En el área de estudio es posible encontrar formas volcánicas, tanto elementales como complejas como son: coladas de lava, domos, calderas volcánicas, cráteres, formas de erosión diferencial, así como un pequeño sector de tipo intrusivo.

Según Denyer y Alvarado (2007) la geología del área de estudio está compuesta por rocas volcánicas del Plio-Pleistoceno y su basamento está compuesto por rocas del Grupo Aguacate, que corresponden con depósitos volcánicos del Mioceno. Sin embargo, estas rocas no afloran en el área de la Reserva.

Según los estudios realizados en el nivel de la sección de la cordillera de Tilarán y que cubren en parte la ReBAMB (Weyl 1956; Dengo 1962; Chaves y Sáenz, 1973, Sprechmann 1984; Vargas, 1991; Villegas, 1997, Vargas, 2001, Denyer y Alvarado, 2007) y de los análisis de fotografías aéreas, se han encontrado que dominan las formaciones Aguacate y Monte Verde, y otras unidades litológicas derivados de ambos, las cuales se analizan a continuación. 


\section{Formación monte verde}

La formación Monteverde sobreyace discordantemente al Grupo Aguacate y está compuesta por espesos depósitos de ignimbritas en la base de la formación, que son sobreyacidos por las andesítico piroxénicas (Cigolini y Chaves, 1986) citado por Vargas (2001). Esta formación ha sido reconocida por formar diques hipoabisales y relictos volcánicos. Los diques andesíticos tienen espesores que varían desde decímetros hasta metros, los cuales se observan en algunos sitios, por ejemplo en ciertos sectores del río San Lorencito (figura 2).

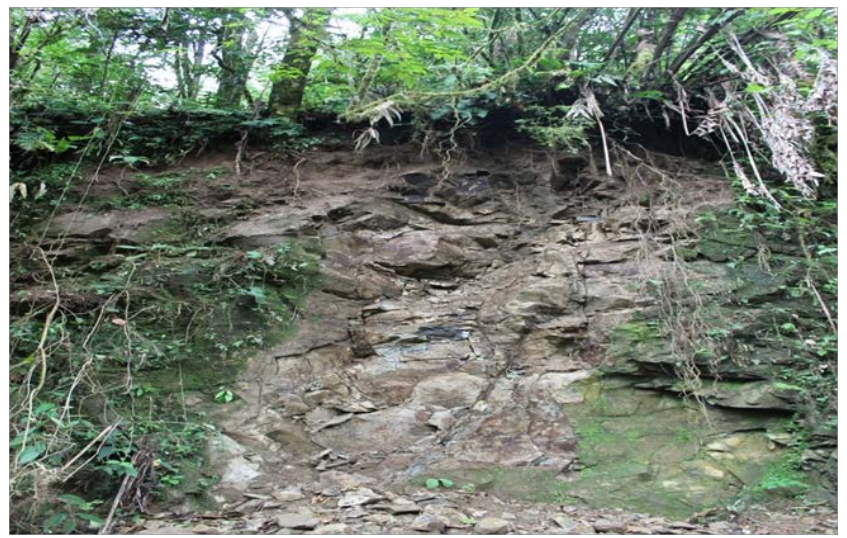

Fig. 2. Rocas basálticas existentes en cortes de caminos y en las márgenes de algunos ríos.

Es posible reconocer edificios volcánicos que están formados por andesita piroxénicas o andesitas con hornblenda y que están bajo un proceso de erosión inicial. Un ejemplo de ello son los cerros Bekon, los cerros Jamaical y Jabonal.

La formación Monteverde se reconoce fácilmente por sus lavas andesíticas, por su posición estratigráfica sobre el Grupo Aguacate y por sus relieves morfológicos menos afectados por la erosión. Los depósitos piroclásticos de la Formación Monteverde se diferencian del Grupo Aguacate por su litología. Dentro de la Formación Monteverde se distinguen dos tipos de formaciones volcánicas muy diferentes. En primera instancia, al norte de la Reserva existe una caldera volcánica donde incluso quedan algunos remanentes como son lagunas. Esta estructura de colapso presenta además la particularidad de ser el sitio con la topografía de menor pendiente existente en la Reserva (figura 3).

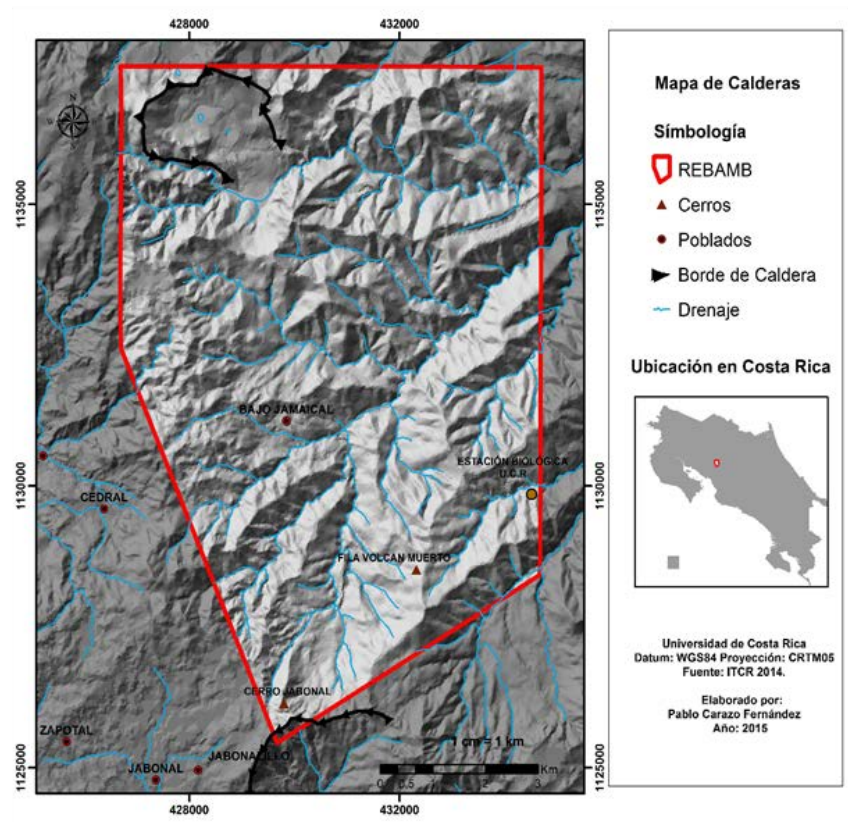

Fig. 3. Calderas volcánicas y principales cerros existentes en la ReBAMB.

Al sur de la Reserva, en el cerro Jabonal que tiene una altitud de 1520 m.s.n.m., se presenta un grupo de relictos volcánicos, caracterizados por poseer la forma de domo andesítico y que cubre una superficie de aproximadamente $8 \mathrm{~km}^{2}$. Dichos relictos volcánicos se ubican en la cuenca de captación de los ríos San Lorenzo, San Lorencito y quebrada González.

La actividad volcánica formó varias geoformas, entre ellas los cráteres producto de las explosiones, situación que fue recalcada por Artavia (2008) que cita la existencia de lagunas cratéricas en Bajo Jamaical. De forma similar, Bergoing (2008) y Veas (2009) señalan la presencia de una caldera volcánica entre la Fila Cedral, el Cerro Jabonal y la Fila Volcán Muerto. De igual forma, Vargas (2001) encontró que no solo existe una similitud topográfica, sino también la existencia de antiguos cráteres en la región norte y noroeste del río Palmital, lo que sugiere que hubo una intensa actividad volcánica.

La región NW, SW y las cadenas montañosas que corren paralelas a los ríos en la cuenca de río San Lorenzo tienen una constitución predominante de coladas de basalto y lavas andesíticas que originaron un relieve de vulcanismo perteneciente 
al Grupo Aguacate (Mioceno Superior). Esta sección volcánica se caracteriza por un sistema montañoso muy irregular, con pendientes abruptas y cañones de ríos profundos, lo que da como resultado la existencia de pendientes que oscilan entre los 25 y 30 grados. Dichas pendientes existentes en sitios como son las nacientes del río San Lorenzo, mesetas de Bajo Jamaical y las depresiones de lagunas cretácicas de la Reserva Biológica Alberto Ml. Brenes (Vargas, 1978).

Asimismo, algunos cañones de los ríos, como es el caso del río San Lorenzo y San Lorencito, muestran coladas de lava basálticas y andesíticas, así como depósitos de lahares que se ubican sobre las primeras. Dichas rocas se encuentran poco alteradas pese a la gran humedad de la zona, lo cual es un indicativo de la resistencia de las rocas y de la edad del vulcanismo (figura 4).

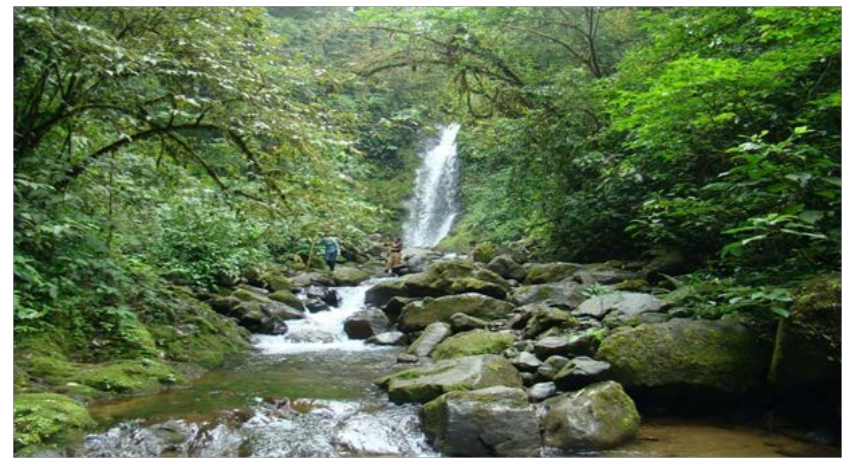

Fig. 4. Afloramientos en el río San Lorencito.

Hacia el NW se da un relieve asociado a formas de depositación lahárica caracterizado por la existencia de domos y lomas con pendientes altas y moderadas. En el extremo NW de la cuenca del río San Lorenzo se dan conglomerados volcánicos $\mathrm{y}$ formas de erosión diferencial que dieron origen a un relieve con pendientes superiores a 15 grados.

A pesar de la densa cobertura forestal que presenta la Reserva, se pueden ver en algunos sectores cenizas volcánicas. Por ejemplo algunas de ellas se observan en cortes de camino, las cuales a su vez son las que más fácilmente se erosionan cuando los suelos quedan descubiertos al ocurrir pequeños deslizamientos o cuando se da la caída de grandes árboles dentro del bosque.

\section{Formación aguacate}

Esta formación se encuentra en la base de la secuencia estratigráfica o subyace la formación Monteverde. Está compuesta por fenobasaltos y fenoandesitas basálticas con brechas lávicas y brechas piroclásticas, en capas de espesores métricas y decamétricas (Vargas, 2001). Según Vargas (2001), las coladas de lava son afaníticas o afanítico porfiríticas, con espesores que varían entre cuatro y diez metros, con abundante contenidos de piroxenos y plagioclasa. El Grupo Aguacate de la Reserva en estudio se caracteriza por esta muy bien definida desde el punto de vista lítico. Se presentan ciertas alteraciones hidrotermales, lo cual se refleja en el alto contenido de arcillas en ciertos sectores. Esto se ha dado como consecuencia de la alta permeabilidad de los suelos y al fallamiento local (figura 5).

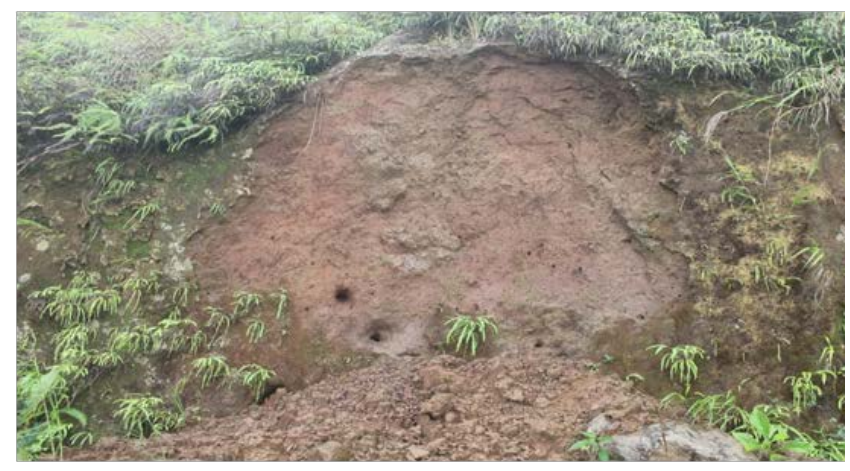

Fig. 5. Afloramiento rocoso de lavas volcánicas con tonos rojizos y grisáceos dado el alto estado de meteorización.

En cortes de camino y algunas paredes de los cauces se encuentran estratos con brechas volcaniclásticas que muestran una condición arcillificada intercaladas con lavas basálticas que muestran un estado de meteorización química elevado, tal como se muestra en la fotografía anterior. Alfaro (2000), describe el Grupo Aguacate como rocas muy alteradas, con tonos verdosos, violáceos y pardos. Define dentro del área del proyecto dos unidades del grupo: la unidad de Basaltos, asociada con las andesitas Miramar y la unidad de Brechas, la cual se asocia con las Brechas La Unión; además, le asigna a estas rocas una edad Paleoceno- Plioceno. 


\section{Otras unidades litológicas}

Veas (2009) en una investigación realizada al SE de la ReBAMB indica que existen coladas de basalto, lavas andesíticas, aglomerados, tobas y brechas de espesor desconocido en forma estratificada que han sido cortadas por diques de basalto. Vargas (1978) y Veas (2009) consideran que dicho sector de la ReBAMB presenta un estado de desarrollo erosional de juventud avanzada, así como un rejuvenecimiento debido a la elevación de un horst.

Existen en la Reserva elevaciones en los cauces de los ríos y márgenes escarpadas, lo cual es producto de coladas de lava expuestas. Además, podría deberse a la fuerte erosión de antiguos conos volcánicos, según Kussmaul (2006) (citado por Veas, 2009). Por consiguiente, se puede inducir que existe la posibilidad de una actividad volcánica reciente, aspecto que fue sustentado por Castillo (1984), quien se basó en el tipo de rocas encontradas y la presencia de un intrusivo en el sector oeste de la Reserva.
De acuerdo con Vargas (1978), una parte de la Reserva está constituida por coladas de basalto, lavas andesíticas, aglomerados, tobas y brechas autoclásicas de espesor desconocido en forma estratificada, que han sido cortadas por diques de basalto, y se da también una mineralización aurífera. Existe además una zona de lahares extensos, los cuales constituyen depósitos de corrientes de barro en aquellas zonas inferiores a 800 m.s.n.m., provenientes de la región montañosa del Grupo Aguacate. Se ubican principalmente en sectores aledaños a la ReBAMB, por ejemplo, al NE de la cuenca, en las poblaciones de Las Rocas, San Jorge y San Lorenzo, en la parte central, de forma que cubre la Colonia Palmareña, así como en el sector que se ubica al sureste de la finca Santa Cecilia hasta El Peñón. Por las características topográficas de la Reserva se puede deducir que hubo un vulcanismo explosivo muy violento al inicio y luego le sigue la extrusión, la cual se observa al existir una serie de formas volcánicas cónica (figura 6).

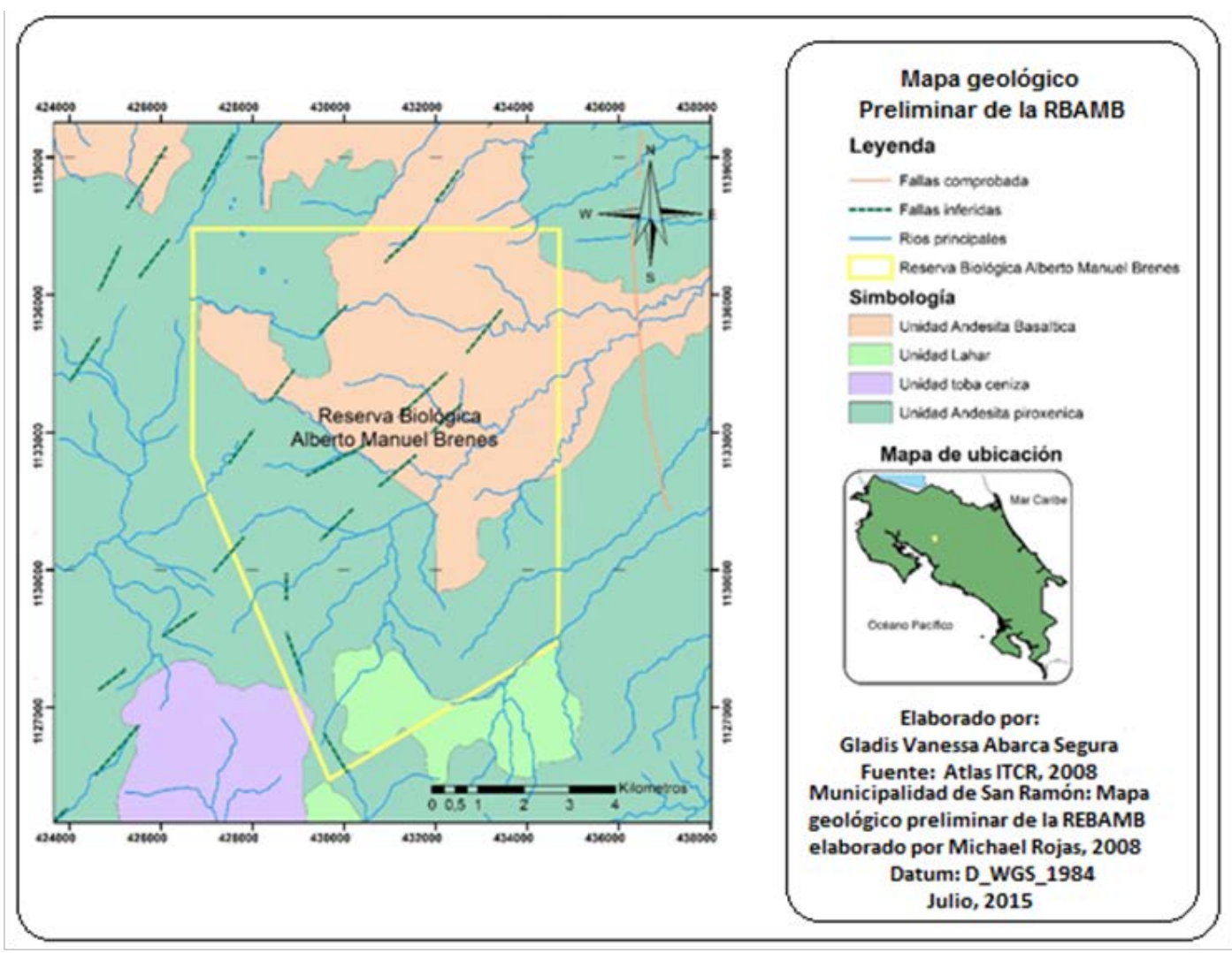

Fig. 6. Mapa Geológico de la Reserva Biológica Alberto Manuel Brenes. 
Según Madrigal (1984) y Alvarado et al. (2000), las rocas volcánicas en este sector que comprende la ReBAMB en gran parte son producto de un volcanismo fisural, muy probablemente originado por un sistema de fallas que se produjo a su vez por el ascenso de los intrusivos. Además, ocurrieron emanaciones de flujos piroclásticos y lávicos fisúrales.

\section{Estructuras tectónicas}

La tectónica regional está caracterizada por sistemas de fallas y de fracturas producto, tanto de los esfuerzos generados por el proceso de subducción de la placa del Coco bajo la placa Caribe, como por los cuerpos intrusivos que conforman la sección de la cordillera de Tilarán. En la zona se observan patrones de fallamiento principales de orientación NE-SE, NE-SW y NNE-SSW. (Vargas, 2001)

En la ReBAMB existen tres grandes fallas geológicas con una dirección NE-SW, incluso dos de estas cruzan completamente la Reserva. La primera de ellas se ubica en la margen izquierda del río Jamaical (Falla Jamaical) y la segunda en la margen derecha del río San Lorenzo, en tanto, la tercera se ubica en la naciente de la quebrada Cacical. En todas ella se nota la existencia de desplazamientos siniéstrales. Los patrones de drenaje están controlados en gran parte por este sistema de fallamiento En la figura 7 se observa un afloramiento ubicado a la entrada de la estación experimental que refleja la ausencia de acción hidrotermal y un intenso lajamiento. Parte de este afloramiento se deslizó durante el terremoto del 5 de septiembre de 2012.

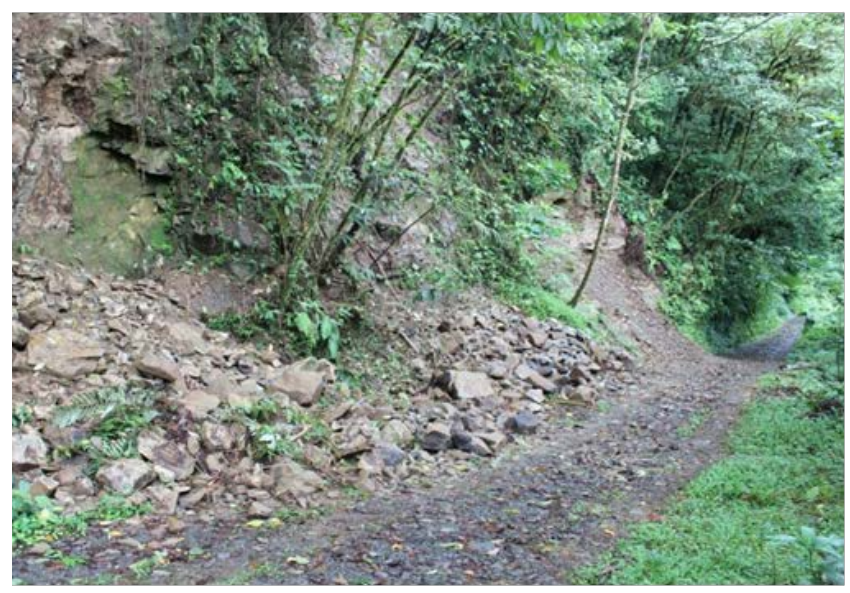

Fig.7. Afloramiento con ausencia de acción hidrotermal.

Bergoing (2007) y Veas (2009) indican que existe una intensa actividad tectónica, lo cual se refleja en el río San Lorencito, que se orienta sobre una falla en su parte media, dado lo anterior, el relieve de la zona montañosa se encuentra muy fracturado y accidentado; se manifiestan diferencias de hasta 300 metros entre las partes montañosas y los cauces de los ríos. De ahí, según Veas (2009) toda la Reserva se caracteriza por tener ríos rodeados de fuertes taludes de erosión.

Denyer y Kussmaul (2000), citados por Veas (2009), sugieren que dada la gran cantidad de fallas en toda el área, los primeros indicios de actividad tectónica fueron de tipo fisural. Estas posiblemente constituyen el material más antiguo, encontrado debajo del lecho del río. Dicho material es de tipo basáltico, el cual no está todavía meteorizado pese a la gran humedad existente en la zona (figura 7). Con respecto a la amenaza sísmica en la zona la reserva, para un período de retorno de 500 años, que es lo que estipula el Código Sísmico de Costa Rica para el diseño sismo resistente, la máxima aceleración sísmica esperable (PGA) en el nivel de lecho rocoso, es del orden de 4.0 a $4.5 \mathrm{~m} / \mathrm{s} 2$ en esa zona (Benito y Torres, 2009). 


\section{Conclusiones}

La geología de la ReBAMB se originó inicialmente por un vulcanismo explosivo, caracterizado por la emanación de flujos lávicos piroclásticos. El área se encuentra constituida principalmente por rocas ígneas y sedimentarias, en su mayor parte terciarias, en tanto que los depósitos cuaternarios son producto de la erosión y meteorización de las rocas más antiguas. Por otro lado, ocurrió la implantación de intrusivos, como es el caso de Guacimal.

En la Reserva se encuentra emplazado el Grupo Aguacate, el cual sirve como basamento geológico y la Formación Monteverde, que ha sido producida por un magmatismo dacítico explosivo y uno posterior vulcanismo andesítico. Las estructuras geológicas presentan formas distintivas, con fallas geológicas posiblemente activas, originadas por las deformaciones al interior de la zona del arco volcánico, que viene a ser el resultado de la subducción de la placa Cocos debajo de la placa Caribe por el levantamiento que producen cuerpos intrusivos. Existen además formas caldéricas, producto de la explosión o colapsos volcánicos.

La topografía de la Reserva muestra las condiciones litológicas propias de la ReBAMB, caracterizada principalmente por aparatos volcánicos con coladas de lava, domos, calderas volcánicas, cráteres y formas de erosión diferencial. Similarmente, existen cuerpos intrusivos granitoides y rocas sedimentarias, localmente meteorizadas, así como lavas de posibles cuerpos subvolcánicos, subordinadas en la secuencia estratigráfica. Por otro lado, gran parte del patrón de drenaje que comprende el sector donde se ubica la Reserva, escurre hacia la vertiente Caribe y solo una pequeña porción del territorio drena hacia la vertiente Pacífica.

Este estudio servirá para establecer no solo políticas y planes de acción para llevarse a cabo en la ReBAMB, por lo que entre los aportes expuestos, es importante considerar el valor de amenaza sísmica aquí sugerido del orden de 4.0 a $4.5 \mathrm{~m} / \mathrm{s}^{2}$ en esa zona. Además, la información expuesta servirá como base para planificar posibles aprovechamientos de agua para fines hidroeléctricos. Por otro lado, este estudio demuestra que la categoría de manejo de reserva biológica es ideal dado que se conservan distintos recursos naturales, gracias a que no es un lugar que sirva para el turismo, sino más bien para la investigación y la educación ambiental. 


\section{Bibliografía}

Alfaro, A. (2000). Clasificación Geomecánica del Macizo Rocoso en la Zona de Momees, Proyecto Bellavista Miramar de Puntarenas. - Tesis Lic. ECG - UCR, 43 p.

Alvarado.G.E. (2000). Volcanes de Costa Rica: geología, historia y riqueza natural. 269 págs. San José: EUNED.

Artavia, L. G. (2008). La evolución de los frentes de colonización agrícola sobre la Reserva Biológica Alberto Manuel Brenes, 1975-2005. Práctica de la Geografía 2. Cartago: Editorial Tecnológica de Costa Rica.

Bergoing, J. P. (1998). Geomorfología de Costa Rica. Instituto Geográfico Nacional. 409 p.

Bergoing J.P. (2007). Geomorfología de Costa Rica. San José: Librería Francesa. 328 págs. (es del 20070 del 2008)

Benito, B. y Torres, Y. (2009). Amenaza sísmica de América Central. Madrid: Editorial Ethinama, $371 \mathrm{p}$.

Bergoing, J. (2008). Geomorfología de Costa Rica. San José: Librería Francesa, 328 p.

Castillo, R. (1984). Geología de Costa Rica: Una sinopsis. San José: Editorial Universidad de Costa Rica. 182 p.

Cortes, V. (1994). Costa Rica: Su evolución geológica está en el fondo del mar. San José: Ed. Guayacán, 56 p.

Chaves, R. y Sáenz, R. (1974): Informe técnico y geológico de la cordillera de Tilarán. Ministerio de Economía, Industria y Comercio. Dirección Geología, Minas y Petrología. San José. CR. $74 \mathrm{p}$.

Denyer, P.; Alvarado, G.E. (2007). Mapa geológico de Costa Rica.- Escala 1:400 000. San José: Librería Francesa.
Dengo, G. (1962). Estudio geológico de la región de Guanacaste, Costa Rica. IGN. Costa Rica. $112 \mathrm{p}$.

Denyer, P.; Kussmaul, S. (2000). Geología de Costa Rica. Cartago: Ed. Tecnológica de Costa Rica. $515 \mathrm{p}$.

Kussmaul, S. (2006). "Petrografía de las rocas Intrusivas neógeno de Costa Rica". Rev. Geología de América Central.7: 83-112 p.

Lloyd, J. (1963). Historia tectónica del orogeno sur centroamericano. Informe Semestral. Ene-Jun. Instituto Geográfico Nacional, Costa Rica. 67-96 p.

Madrigal y Rojas. (1984). Mapa geomorfológico de Costa Rica, 9 mapas, Escala 1: 200000. San José: Imprenta Nacional. SEPSA.

Salazar, A. (2000). Reserva Biológica Alberto Manuel Brenes: Veinticinco años de conservación, investigación y bioalfabetización. Universidad de Costa Rica. 74 p.

Veas, M. (2009): Caracterización y estimación de la erosión laminar en un bosque premontano a partir de un modelado hidrológico. Microcuenca del río San Lorencito, Cordillera Volcánica de Tilarán, Costa Rica. Tesis de Licenciatura en Geología. Universidad de Costa Rica. 132 p.

Vargas, G. (1978). Diagnostico y recomendaciones para el manejo y ordenamiento de los recursos naturales en la cuenca del río San Lorenzo, Alajuela, Costa Rica. Tesis licenciatura en Geografía. UCR. San José, CR. 132 p.

Vargas, J. (2001). Geología, hidrogeoquímica y modelo conceptual de reservorio para la prefactibilidad del campo geotérmico Poco Sol, San Ramón, San Carlos, Costa Rica. Tesis Programa de posgrado en Geología. Universidad de Costa Rica. 132 p.

Weyl, R. (1956). Vulcanismo y plutonismo en el sur de Centroamérica. Informe Trimestral Jul-Set. Instituto Geográfico Nacional, Costa Rica. 55 p. 
